\title{
COPULA-BASED STATISTICAL MODELLING OF SYNOPTIC-SCALE CLIMATE INDICES FOR QUANTIFYING AND MANAGING AGRICULTURAL RISKS IN AUSTRALIA
}

\author{
THONG NGUYEN-HUY ${ }^{\circledR}$
}

(Received 15 July 2019; first published online 25 November 2019)

2010 Mathematics subject classification: primary 62H12; secondary 62P05, 62P12, $68 \mathrm{~T} 10$.

Keywords and phrases: multivariate analysis, copula, climate, agricultural risk, insurance.

Australia is an agricultural nation characterised by one of the most naturally diverse climates in the world, which translates into significant sources of risk for agricultural production and subsequent farm revenues. Extreme climatic events have been significantly affecting large parts of Australia in recent decades, contributing to an increase in the vulnerability of crops, and leading to subsequent higher risk to a large number of agricultural producers. However, attempts at better management of climate-related risks in the agricultural sector have confronted many challenges.

First, crop insurance products, including classical claim-based and index-based insurance, are among the financial implements that allow exposed individuals to pool resources to spread their risk. The classical claim-based insurance indemnifies according to a claim of crop loss from the insured customer and so can easily manage idiosyncratic risk, which is the case where the loss occurs independently. Nevertheless, the existence of systemic weather risk (covariate risk), which is the spread of extreme events over locations and times (for example, droughts and floods), has been identified as the main reason for the failure of private insurance markets, such as the classical multiperil crop insurance, for agricultural crops. The index-based insurance is appropriate to handle systemic but not idiosyncratic risk. The indemnity payments of the index-based insurance are triggered by a predefined threshold of an index (for example, rainfall), which is related to such losses. Because of the covariate nature of a climatic event, it sanctions the insurers to predict losses and ascertain indemnifications for a huge number of insured customers across a wide geographical area. However, basis risk, which is related to the strength of the relationship between

Thesis submitted to the University of Southern Queensland in August 2018; degree approved on 11 February 2019; principal supervisor Ravinesh Deo, associate supervisors Shahbaz Mushtaq and Shahjahan Khan.

(C) 2019 Australian Mathematical Publishing Association Inc. 
the predefined indices used to estimate the average loss by the insured community and the actual loss of insured assets by an individual, is a major barrier that hinders uptake of the index-based insurance. Clearly, the high basis risk, which is a weak relationship between the index and loss, destroys the willingness of potential customers to purchase this insurance product.

Second, the impact of multiple synoptic-scale climate mode indices (for example, southern oscillation index (SOI) and Indian Ocean index (IOD)) on precipitation and crop yield is not identical in different spatial locations and at different times or seasons across the Australian continent since the influence of large-scale climate is heterogeneous over the different regions. The occurrence, role and amplitude of synoptic-scale climate modes contributing to the variability of seasonal crop production have shifted in recent decades. These variables generally complicate the climate and crop yield relationship that cannot be captured by traditional modelling and analysis approaches commonly found in published agronomic literature, such as linear regression. In addition, the traditional linear analysis is not able to model the nonlinear and asymmetric interdependence between extreme insurance losses, which may occur in the case of systemic risk. Relying on the linear method may lead to the problem that different behaviour may be observed from joint distributions, particularly in the upper and lower regions, with the same correlation coefficient. As a result, the likelihood of extreme insurance losses can be underestimated or overestimated, leading to inaccuracies in the pricing of insurance policies. Another alternative is the use of the multivariate normal distribution, where the joint distribution is uniquely defined using the marginal distributions of variables and their correlation matrix. However, phenomena are not always normally distributed in practice.

It is therefore important to develop new, scientifically verified, strategic measures to solve the challenges mentioned above and mitigate the influences of the climaterelated risk in the agricultural sector. Copulas provide an advanced statistical approach to model the joint distribution of multivariate random variables. This technique allows estimation of the marginal distributions of individual variables independently of their dependence structures. It is clear that the copula method is superior to conventional linear regression since it does not require variables to be normally distributed and their correlation can be either linear or nonlinear.

This doctoral thesis therefore adopts the advanced copula technique within a statistical modelling framework that aims to model: (1) the compound influence of synoptic-scale climate indices (that is, SOI and IOD) and climate variables (that is, precipitation) to develop a probabilistic precipitation forecasting system where the integrated role of different factors that govern precipitation dynamics are considered; (2) the compound influence of synoptic-scale climate indices on wheat yield; (3) the scholastic interdependencies of systemic weather risks where potential adaptation strategies are evaluated accordingly; and (4) the risk-reduction efficiencies of geographical diversifications in wheat-farming portfolio optimisation. The study areas are Australia's agroecological (that is, wheat belt) zones where major seasonal wheat and other cereal crops are grown. The results from the first and second objectives 
can be used not only for forecasting purposes but also for understanding the basis risk in the case of pricing climate index-based insurance products. The third and fourth objectives assess the interactions of drought events across different locations and in different seasons, with different feasible adaptation tools. The findings of these studies can provide useful information for decision-makers in the agricultural sector.

The first study found a significant relationship between SOI, IOD and precipitation. The results suggest that spring precipitation in Australia, except for the western part, can be forecast probabilistically three months ahead. It is more interesting that the combination of SOI and IOD as the predictors improves the performance of the forecast model. Similarly, the second study indicated that the large-scale climate indices could provide knowledge of wheat crops up to six months in advance. However, the influence of different climate indices varies over locations and times. The findings derived from the third study demonstrated the spatiotemporally stochastic dependence of the drought events. The results also prove that time diversification is potentially more effective in reducing the systemic weather risk compared with a spatially diversifying strategy. Finally, the fourth objective revealed that a wheatfarming portfolio could be effectively optimised through geographical diversification.

The outcomes of this study will lead to new applications of advanced statistical tools that provide a better understanding of the compound influence of synopticscale climatic conditions on seasonal precipitation, and therefore on wheat crops, in key regions over the Australian continent. A comprehensive analysis of systemic weather risks performed through advanced copula-statistical models can help improve and develop novel agricultural adaptation strategies in not only the selected study region but also globally, where extreme climate events pose a serious threat to the sustainability and survival of the agricultural industry. Finally, the evaluation of the effectiveness of the diversification strategies implemented in this study reveals new evidence on whether the risk-pooling methods could potentially mitigate climate risks for the agricultural sector and help farmers prepare for uncertain climatic events.

Some of this research has been published in [1-4].

\section{References}

[1] T. Nguyen-Huy, R. C. Deo, D. An-Vo, S. Mushtaq and S. Khan, 'Copula-statistical precipitation forecasting model in Australia's agro-ecological zones', Agric. Water Manag. 191 (2017), 153-172.

[2] T. Nguyen-Huy, R. C. Deo, S. Mushtaq, D. An-Vo and S. Khan, 'Modeling the joint influence of multiple synoptic-scale, climate mode indices on Australian wheat yield using a vine copula-based approach', Eur. J. Agron. 98 (2018), 65-81.

[3] T. Nguyen-Huy, R. C. Deo, S. Mushtaq, J. Kath and S. Khan, 'Copula-based agricultural conditional value-at-risk modelling for geographical diversifications in wheat farming portfolio management', Weather Clim. Extremes 21 (2018), 76-89.

[4] T. Nguyen-Huy, R. C. Deo, S. Mushtaq, J. Kath and S. Khan, 'Copula statistical models for analyzing stochastic dependencies of systemic drought risk and potential adaptation strategies', Stoch. Environ. Res. Risk Assess. 33 (2019), 779-799. 
THONG NGUYEN-HUY, Centre for Applied Climate Sciences, University of Southern Queensland, Toowoomba, Queensland 4350, Australia e-mail: thonghuy.nguyen@usq.edu.au 